\title{
Antibiotic resistance changes in strains of bacteria and yeast-like fungi following their growth in established cell lines of human and animal origin
}

\author{
S. L. Rybalko, Ye. V. Pokas, V. A. Dieyev, T. M. Liaskovski, T. M. Furzikova, \\ S. T. Diadiun, N. V. Ivanskaya, N. I. Nastoyashcha, P. P. Verkhatsky, S. T. Salkov
}

Gromashevsky Institute of Epidemiology and Infectious Diseases, Academy of Medical Sciences of Ukraine Acad. Amosov sir., 5, Kyiv, 03038, Ukraine

ginnna@rumbler.ru

\begin{abstract}
The phenomenon of the susceptibility and reversion of resistance to antibiotics in strains of bacteria and yeast-like molds has been identified upon the interaction of bacteria and molds with transplantable human and animal cell lines depending on the cultivation conditions. The ability of susceptibility reversion of bacteria and yeast-like fungi to some antibiotics is not stable, it varies with the passages. This phenomenon may be a reason of the poor efficacy of antibiotic treatment when the susceptibility of bacteria to antibiotics is assayed by the usual technique. The authors propose to modify the antibiotics-susceptibility assay currently used in clinics by co-culture of the isolated bacteria and fungi with the transplantable cell lines or human leukocytes.
\end{abstract}

Keywords: antibiotics, cells, enterobacteria, resistance.

Introduction. Bacterial and yeast-like organisms are usually isolated using nutrient media; the same media are taken to verify the antibiotic resistance of these microorganisms. However, a lot of different strains of bacteria and yeast-like organisms are parasites inhabiting different human and animal organs consisting of different type cells (epithelium-like, fibroblast-like, etc.). There are some published data [1] describing the adhesion process properties during shigellae interactions with human embryo intestinal mucosa.

Bacteria colonize the biomaterial, thereby adopting a sessile mode of growth that progresses to the establishment of an antibiotic-resistant biofilm by the accretion of prospective glycocalyx $[2,3]$. Amino- glycosides, quinolones, beta-lactams, and polymixins require crossing the bacterial cell wall to reach their targets. Penetration can be reduced by the development of antibiotics-resistant biofilms with secretion of an anionic exopolysaccaride matrix with binds cationic antibiotic [6 ]; quorum sensing in such biofilms may also be involved in changing bacterial metabolism and reducing sensitivity to antimicrobials $[4-6]$.

Due to this fact, we became interested in the cultivation of bacteria and yeast-like organisms in cells of humans and animals of different species as well as in host-parasite cell interactions and in the antibiotic susceptibility of infectious agents.

Materiais and Methods. Struins of microorganisms. In this investigation, we used the following strains isolated previously in our laboratory: 9 Staphylococcus aureus strains and 2 Staph. epidermidis ones (gram-positive bacteria); 7 Pseudomonas aeruginosa 
strains and 22 different Enterococcus strains (gramnegative bacteria); 17 representatives of the Candida genus and 2 Cryptococcus neoformans strains. Established cell lines taken for these studies were: L41 (human lymphoblastoid cell line), OH-1 (murine lymphoblastoid cell line), RGNR (cell line originated from rat Gasser node neurinoma), and Vero (green African monkey kidney cell line). Cell cultures were grown in the RPMI-1640 supplemented both with calf foetal serum $(10 \%)$ and antibiotics [7].

We also manipulated with human leukocyte suspension cultures (blood group 0). The cultivation of bacterial strains was performed as follows: $100 \mu 1$ of bacterial suspension containing $1 \cdot 10^{8}$ cells were added to a leukocyte culture $\left(3 \cdot 10^{6}\right.$ cells $\left./ \mathrm{ml}\right)$. Following bacterial growth during $24 \mathrm{~h}, 100 \mu \mathrm{l}$ of infected leukocyte suspension of the same concentration were transferred into a new leukocyte culture to carry out the $2^{\text {nd }}$ passage. The $3^{\text {rd }}$ one was made similarly. Antibiotic sensitivity of bacterial cultures for each passage was studied and compared with its original sensitivity to antibiotics. In these experiments we used two interferon (IFN) preparations - IFN $-\alpha-2 b$ (Intron, $3 \cdot 10^{6}$ I. U., Shering-Plau, USA), and IFN- $\gamma$ $\left(10^{6}\right.$ I. U., product of the Ukrainian Academy of Agriculture).

Cultivation of bacteria and fungi. Each microbial or fungal strain was grown in cultured $24 \mathrm{~h}$-old monolayer cell lines or in suspended human leukocytes.

Each microbial/fungal suspension $\left(10^{8}\right.$ cells $\left./ \mathrm{ml}\right)$ was put into cell cultures or human leukocyte suspension and cultivated during $24 \mathrm{~h}$ in RPMI-1640 without calf foetal serum and antibiotics. The degree of susceptibility of microbial/fungal strains to antibiotics was determined before their growth in eukaryotic cells grown in the Mueller-Hinton medium (initial susceptibility, IS) and following their cultivation (post-cultivation susceptibility, PCS).

The antibiotic susceptibility was evaluated by the Bauer-Kirby approach (a variant of the disk-diffusion method) [8 ]; all bacterial/fungal strains were grown on the Mueller-Hinton medium; commercial disks used were produced by the (St. Petersburg, Russian Federation). The disk quality control with the Mueller-Hinton medium and different antibiotics was performed each time as well as with any new disk series of nutrient media series. The criteria of their adequacy were verified according to the zone diameter of inhibited growth for etalon strains - Escherichia coli 25922, S. aureus ATCC 25923, and P. aeruginosa ATCC 27853 received from the Alive Culture Museum of our Institute.

The manufacturer's criteria were used to evaluate the efficacy of antibiotics against microbial/fungal agents. Taking into consideration the zone diameter of inhibited growth for investigated microorganisms, the strains were divided into three groups - susceptible strains (SS), moderately resistant strains (MRS), and strains highly resistant to antibiotic(s) (HRS) [9]. We determined antibiotic-susceptible/resistant properties of strains with the following compounds widely used in up-to-date clinical practice:

$\begin{array}{lc}\text { Name of antibiotic } & \text { Its abbreviation } \\ \text { Amikacin } & \text { amk } \\ \text { Amoxicillin } & \text { amx } \\ \text { Amphotericin } & \text { amph } \\ \text { Ampicillin } & \text { amp } \\ \text { Carbenicillin } & \text { car } \\ \text { Cefazolin } & \mathrm{kf} \\ \text { Cefepime } & \mathrm{cep} \\ \text { Cefoperazone } & \mathrm{cpr} \\ \text { Cefotaxime } & \mathrm{ctx} \\ \text { Ceftazidime } & \text { caz } \\ \text { Ceftriaxone } & \text { cro } \\ \text { Chloramphenicol } & \text { chl } \\ \text { Ciprofloxacin } & \text { cip } \\ \text { Clindamycin } & \text { cly } \\ \text { Clotrimazole } & \text { clo } \\ \text { Erythromycin } & \text { ery } \\ \text { Fluconazole } & \text { flu } \\ \text { Gentamicin } & \text { gen } \\ \text { Imipenem } & \text { imp } \\ \text { Oxacillin } & \text { oxa } \\ \text { Rifampin } & \text { rif } \\ \text { Tetracycline } & \text { tet } \\ \text { Tobramycin } & \text { tob }\end{array}$

Results and Discussion. Our data concerning the Enterobacteriaceae susceptibility to antibiotics are given in Table 1.

The analysis of the data presented in Table 1 shows that the Enterobacteriaceae cultivation in the L41 cell line causes the development of two phenotypes: some antibiotic-sensitive strains become resistant ones and vice versa. This Table proves the enterobacterial strains have not changed their susceptibility to gentamicin, cefoperazone, and imipe- 
Table 1

Antibiotic susceptibility of enterobacterial strains grown in the L4I culture during $24 \mathrm{~h}$

\begin{tabular}{|c|c|c|c|c|c|c|c|c|c|c|c|c|c|c|c|}
\hline \multirow{2}{*}{ N Siraits } & \multicolumn{15}{|c|}{ Antibiotic (the zone diameter, mm, of inhibited growth for elalon strains) } \\
\hline & amp & tet & gen & tob & amk & chi & cip & $\mathrm{ctx}$ & $\mathrm{cpr}$ & kí & cro & $\operatorname{caz}$ & $\operatorname{amx}$ & $\operatorname{imp}$ & cep \\
\hline $\begin{array}{l}396 \text { initia- } \\
\text { tion L41 }\end{array}$ & $\begin{array}{l}\text { HRS } \\
\text { HRS }\end{array}$ & $\begin{array}{l}\text { HRS } \\
\text { HRS }\end{array}$ & -- & $\begin{array}{l}\text { HRS } \\
\text { HRS }\end{array}$ & $\begin{array}{l}\text { MRS } \\
\text { HRS }\end{array}$ & $\begin{array}{l}\text { HRS } \\
\text { HRS }\end{array}$ & $\begin{array}{l}\text { MRS } \\
\text { HRS }\end{array}$ & $\begin{array}{l}\text { MRS } \\
\text { HRS }\end{array}$ & - & $\begin{array}{l}\text { HRS } \\
\text { HRS }\end{array}$ & $\begin{array}{l}\text { MRS } \\
\text { HRS }\end{array}$ & $\begin{array}{l}\text { SS } \\
\text { SS }\end{array}$ & $\begin{array}{l}\text { HRS } \\
\text { HRS }\end{array}$ & $\begin{array}{l}\text { SS } \\
\text { SS }\end{array}$ & - \\
\hline $\begin{array}{l}727 \text { initia- } \\
\text { tion } \mathrm{L} 41\end{array}$ & $\begin{array}{l}\text { HRS } \\
\text { HRS }\end{array}$ & $\begin{array}{l}\text { HRS } \\
\text { HRS }\end{array}$ & - & $\begin{array}{l}\text { HRS } \\
\text { HRS }\end{array}$ & $\begin{array}{l}\text { HRS } \\
\text { HRS }\end{array}$ & $\begin{array}{c}\text { SS } \\
\text { HRS }\end{array}$ & $\begin{array}{c}\text { SS } \\
\text { HRS }\end{array}$ & $\begin{array}{c}\text { SS } \\
\text { HRS }\end{array}$ & - & $\begin{array}{c}\text { SS } \\
\text { HRS }\end{array}$ & $\begin{array}{c}\text { SS } \\
\text { HRS }\end{array}$ & $\begin{array}{c}\text { SS } \\
\text { HRS }\end{array}$ & $\begin{array}{c}\text { SS } \\
\text { HRS }\end{array}$ & $\begin{array}{l}\text { SS } \\
\text { SS }\end{array}$ & - \\
\hline $\begin{array}{l}312 \text { initia- } \\
\text { tion } \mathrm{L} 41\end{array}$ & $\begin{array}{l}\text { HRS } \\
\text { HRS }\end{array}$ & $\begin{array}{l}\text { HRS } \\
\text { HRS }\end{array}$ & - & $\begin{array}{l}\text { HRS } \\
\text { HRS }\end{array}$ & $\begin{array}{l}\text { MRS } \\
\text { HRS }\end{array}$ & $\begin{array}{l}\text { HRS } \\
\text { HRS }\end{array}$ & $\begin{array}{l}\text { HRS } \\
\text { HRS }\end{array}$ & $\begin{array}{l}\text { HRS } \\
\text { HRS }\end{array}$ & - & $\begin{array}{l}\text { HRS } \\
\text { HRS }\end{array}$ & $\begin{array}{l}\text { HRS } \\
\text { HRS }\end{array}$ & $\begin{array}{l}\text { MRS } \\
\text { HRS }\end{array}$ & $\begin{array}{l}\text { HRS } \\
\text { HRS }\end{array}$ & $\begin{array}{l}\text { SS } \\
\text { SS }\end{array}$ & - \\
\hline $\begin{array}{l}705 \text { initia- } \\
\text { tion L } 41\end{array}$ & $\begin{array}{l}\text { MRS } \\
\text { HRS }\end{array}$ & $\begin{array}{c}\text { HRS } \\
\text { SS }\end{array}$ & - & $\begin{array}{l}\text { HRS } \\
\text { MRS }\end{array}$ & $\begin{array}{c}\text { HRS } \\
\text { SS }\end{array}$ & $\begin{array}{l}\text { HRS } \\
\text { HRS }\end{array}$ & $\begin{array}{c}\text { SS } \\
\text { MRS }\end{array}$ & $\begin{array}{l}\text { SS } \\
\text { SS }\end{array}$ & - & $\begin{array}{c}\text { HRS } \\
\text { SS }\end{array}$ & $\begin{array}{c}\text { SS } \\
\text { MRS }\end{array}$ & $\begin{array}{l}\text { SS } \\
\text { SS }\end{array}$ & $\begin{array}{c}\text { SS } \\
\text { MRS }\end{array}$ & $\begin{array}{l}\text { SS } \\
\text { SS }\end{array}$ & - \\
\hline $\begin{array}{l}598 \text { initia- } \\
\text { tion } \mathrm{L} 41\end{array}$ & $\begin{array}{l}\text { HRS } \\
\text { HRS }\end{array}$ & $\begin{array}{l}\text { HRS } \\
\text { HRS }\end{array}$ & - & $\begin{array}{l}\text { HRS } \\
\text { HRS }\end{array}$ & $\begin{array}{c}\text { SS } \\
\text { HRS }\end{array}$ & $\begin{array}{l}\text { HRS } \\
\text { HRS }\end{array}$ & $\begin{array}{l}\text { HRS } \\
\text { HRS }\end{array}$ & $\begin{array}{c}\text { SS } \\
\text { HRS }\end{array}$ & - & $\begin{array}{l}\text { HRS } \\
\text { HRS }\end{array}$ & $\begin{array}{c}\text { SS } \\
\text { HRS }\end{array}$ & $\begin{array}{c}\text { SS } \\
\text { HRS }\end{array}$ & $\begin{array}{l}\text { HRS } \\
\text { HRS }\end{array}$ & $\begin{array}{l}\text { SS } \\
\text { SS }\end{array}$ & - \\
\hline $\begin{array}{l}326-1 \text { ini- } \\
\text { tiation L41 }\end{array}$ & $\begin{array}{l}\text { HRS } \\
\text { HRS }\end{array}$ & $\begin{array}{l}\text { HRS } \\
\text { HRS }\end{array}$ & - & $\begin{array}{l}\text { HRS } \\
\text { HRS }\end{array}$ & $\begin{array}{l}\text { MRS } \\
\text { HRS }\end{array}$ & $\begin{array}{l}\text { HRS } \\
\text { HRS }\end{array}$ & $\begin{array}{l}\text { HRS } \\
\text { HRS }\end{array}$ & $\begin{array}{l}\text { MRS } \\
\text { HRS }\end{array}$ & - & $\begin{array}{l}\text { HRS } \\
\text { HRS }\end{array}$ & $\begin{array}{l}\text { MRS } \\
\text { HRS }\end{array}$ & $\begin{array}{c}\text { SS } \\
\text { MRS }\end{array}$ & $\begin{array}{l}\text { MRS } \\
\text { HRS }\end{array}$ & $\begin{array}{l}\text { SS } \\
\text { SS }\end{array}$ & - \\
\hline $\begin{array}{l}527 \text { initia- } \\
\text { tion L41 }\end{array}$ & $\begin{array}{l}\text { HRS } \\
\text { HRS }\end{array}$ & $\begin{array}{l}\text { HRS } \\
\text { HRS }\end{array}$ & - & $\begin{array}{l}\text { HRS } \\
\text { HRS }\end{array}$ & $\begin{array}{l}\text { MRS } \\
\text { HRS }\end{array}$ & $\begin{array}{l}\text { HRS } \\
\text { HRS }\end{array}$ & $\begin{array}{c}\text { SS } \\
\text { HRS }\end{array}$ & $\begin{array}{l}\text { SS } \\
\text { SS }\end{array}$ & - & $\begin{array}{l}\text { HRS } \\
\text { HRS }\end{array}$ & $\begin{array}{l}\text { SS } \\
\text { SS }\end{array}$ & $\begin{array}{l}\text { SS } \\
\text { SS }\end{array}$ & $\begin{array}{l}\text { HRS } \\
\text { HRS }\end{array}$ & $\begin{array}{l}\text { SS } \\
\text { SS }\end{array}$ & - \\
\hline $\begin{array}{l}1551-1 \text { ini- } \\
\text { tiation L41 }\end{array}$ & $\begin{array}{l}\text { SS } \\
\text { SS }\end{array}$ & $\begin{array}{l}\text { SS } \\
\text { SS }\end{array}$ & $\begin{array}{l}\text { SS } \\
\text { SS }\end{array}$ & $\begin{array}{l}\text { SS } \\
\text { SS }\end{array}$ & $\begin{array}{l}\text { SS } \\
\text { SS }\end{array}$ & $\begin{array}{l}\text { SS } \\
\text { SS }\end{array}$ & $\begin{array}{l}\text { SS } \\
\text { SS }\end{array}$ & $\begin{array}{l}\text { SS } \\
\text { SS }\end{array}$ & $\begin{array}{l}\text { SS } \\
\text { SS }\end{array}$ & $\begin{array}{l}\text { HRS } \\
\text { SS }\end{array}$ & $\begin{array}{l}\text { SS } \\
\text { SS }\end{array}$ & $\begin{array}{l}\text { SS } \\
\text { SS }\end{array}$ & $\begin{array}{l}\text { SS } \\
\text { SS }\end{array}$ & $\begin{array}{l}\text { SS } \\
\text { SS }\end{array}$ & $\begin{array}{l}\text { SS } \\
\text { SS }\end{array}$ \\
\hline $\begin{array}{l}1551-2 \text { ini- } \\
\text { tiation } \mathrm{L} 41\end{array}$ & $\begin{array}{l}\text { HRS } \\
\text { HRS }\end{array}$ & $\begin{array}{l}\text { HRS } \\
\text { HRS }\end{array}$ & $\begin{array}{l}\text { SS } \\
\text { SS }\end{array}$ & $\begin{array}{l}\text { MRS } \\
\text { SS }\end{array}$ & $\begin{array}{l}\text { SS } \\
\text { SS }\end{array}$ & $\begin{array}{l}\text { SS } \\
\text { SS }\end{array}$ & $\begin{array}{c}\text { HRS } \\
\text { SS }\end{array}$ & $\begin{array}{l}\text { SS } \\
\text { SS }\end{array}$ & $\begin{array}{l}\text { SS } \\
\text { SS }\end{array}$ & $\begin{array}{c}\text { MRS } \\
\text { SS }\end{array}$ & $\begin{array}{l}\text { SS } \\
\text { SS }\end{array}$ & $\begin{array}{l}\text { SS } \\
\text { SS }\end{array}$ & $\begin{array}{l}\text { MRS } \\
\text { MRS }\end{array}$ & $\begin{array}{l}\text { SS } \\
\text { SS }\end{array}$ & $\begin{array}{l}\text { SS } \\
\text { SS }\end{array}$ \\
\hline $\begin{array}{l}1532-1 \text { ini- } \\
\text { tiation } \mathrm{L} 41\end{array}$ & $\begin{array}{l}\text { HRS } \\
\text { HRS }\end{array}$ & $\begin{array}{l}\text { HRS } \\
\text { HRS }\end{array}$ & $\begin{array}{l}\text { HRS } \\
\text { HRS }\end{array}$ & $\begin{array}{l}\text { HRS } \\
\text { HRS }\end{array}$ & $\begin{array}{l}\text { HRS } \\
\text { MRS }\end{array}$ & $\begin{array}{l}\text { SS } \\
\text { SS }\end{array}$ & $\begin{array}{l}\text { HRS } \\
\text { HRS }\end{array}$ & $\begin{array}{l}\text { HRS } \\
\text { HRS }\end{array}$ & $\begin{array}{l}\text { HRS } \\
\text { HRS }\end{array}$ & $\begin{array}{l}\text { HRS } \\
\text { HRS }\end{array}$ & $\begin{array}{l}\text { HRS } \\
\text { HRS }\end{array}$ & $\begin{array}{l}\text { HRS } \\
\text { HRS }\end{array}$ & $\begin{array}{l}\text { HRS } \\
\text { HRS }\end{array}$ & $\begin{array}{l}\text { SS } \\
\text { SS }\end{array}$ & $\begin{array}{l}\text { MRS } \\
\text { MRS }\end{array}$ \\
\hline $\begin{array}{l}1532-2 \text { ini- } \\
\text { tiation L41 }\end{array}$ & $\begin{array}{l}\text { HRS } \\
\text { HRS }\end{array}$ & $\begin{array}{l}\text { HRS } \\
\text { HRS }\end{array}$ & $\begin{array}{l}\text { SS } \\
\text { SS }\end{array}$ & $\begin{array}{l}\text { MRS } \\
\text { SS }\end{array}$ & $\begin{array}{c}\text { SS } \\
\text { MRS }\end{array}$ & $\begin{array}{l}\text { SS } \\
\text { SS }\end{array}$ & $\begin{array}{l}\text { SS } \\
\text { SS }\end{array}$ & $\begin{array}{l}\text { SS } \\
\text { SS }\end{array}$ & $\begin{array}{l}\text { SS } \\
\text { SS }\end{array}$ & $\begin{array}{l}\text { MRS } \\
\text { MRS }\end{array}$ & $\begin{array}{l}\text { SS } \\
\text { SS }\end{array}$ & $\begin{array}{l}\text { SS } \\
\text { SS }\end{array}$ & $\begin{array}{l}\text { SS } \\
\text { HRS }\end{array}$ & $\begin{array}{l}\text { SS } \\
\text { SS }\end{array}$ & $\begin{array}{l}\text { SS } \\
\text { SS }\end{array}$ \\
\hline $\begin{array}{l}1532-3 \text { ini- } \\
\text { tiation } \mathrm{L} 41\end{array}$ & $\begin{array}{l}\text { HRS } \\
\text { HRS }\end{array}$ & $\begin{array}{l}\text { HRS } \\
\text { MRS }\end{array}$ & $\begin{array}{l}\text { SS } \\
\text { SS }\end{array}$ & $\begin{array}{l}\text { HRS } \\
\text { SS }\end{array}$ & $\begin{array}{l}\text { MRS } \\
\text { SS }\end{array}$ & $\begin{array}{l}\text { SS } \\
\text { SS }\end{array}$ & $\begin{array}{l}\text { SS } \\
\text { SS }\end{array}$ & $\begin{array}{l}\text { SS } \\
\text { SS }\end{array}$ & $\begin{array}{l}\text { SS } \\
\text { SS }\end{array}$ & $\begin{array}{c}\text { HRS } \\
\text { SS }\end{array}$ & $\begin{array}{l}\text { SS } \\
\text { SS }\end{array}$ & $\begin{array}{l}\text { SS } \\
\text { SS }\end{array}$ & $\begin{array}{c}\text { SS } \\
\text { HRS }\end{array}$ & $\begin{array}{l}\text { SS } \\
\text { SS }\end{array}$ & $\begin{array}{l}\text { SS } \\
\text { SS }\end{array}$ \\
\hline $\begin{array}{l}1004 \text { ini- } \\
\text { tiation L41 }\end{array}$ & $\begin{array}{l}\text { HRS } \\
\text { HRS }\end{array}$ & $\begin{array}{l}\text { HRS } \\
\text { HRS }\end{array}$ & $\begin{array}{l}\text { HRS } \\
\text { HRS }\end{array}$ & $\begin{array}{l}\text { HRS } \\
\text { HRS }\end{array}$ & $\begin{array}{c}\text { SS } \\
\text { HRS }\end{array}$ & $\begin{array}{l}\text { SS } \\
\text { SS }\end{array}$ & $\begin{array}{l}\text { MRS } \\
\text { HRS }\end{array}$ & $\begin{array}{l}\text { SS } \\
\text { SS }\end{array}$ & $\begin{array}{l}\text { SS } \\
\text { SS }\end{array}$ & $\begin{array}{l}\text { MRS } \\
\text { MRS }\end{array}$ & $\begin{array}{l}\text { SS } \\
\text { SS }\end{array}$ & $\begin{array}{l}\text { SS } \\
\text { HRS }\end{array}$ & $\begin{array}{l}\text { HRS } \\
\text { HRS }\end{array}$ & $\begin{array}{l}\text { SS } \\
\text { SS }\end{array}$ & $\begin{array}{l}\text { HRS } \\
\text { MRS }\end{array}$ \\
\hline $\begin{array}{l}963 \text { initia- } \\
\text { tion L41 }\end{array}$ & $\begin{array}{l}\text { HRS } \\
\text { HRS }\end{array}$ & $\begin{array}{l}\text { HRS } \\
\text { HRS }\end{array}$ & $\begin{array}{l}\text { HRS } \\
\text { HRS }\end{array}$ & $\begin{array}{l}\text { HRS } \\
\text { HRS }\end{array}$ & $\begin{array}{l}\text { MRS } \\
\text { MRS }\end{array}$ & $\begin{array}{l}\text { HRS } \\
\text { HRS }\end{array}$ & $\begin{array}{l}\text { HRS } \\
\text { HRS }\end{array}$ & $\begin{array}{l}\text { HRS } \\
\text { HRS }\end{array}$ & $\begin{array}{l}\text { HRS } \\
\text { HRS }\end{array}$ & $\begin{array}{l}\text { HRS } \\
\text { HRS }\end{array}$ & $\begin{array}{l}\text { HRS } \\
\text { HRS }\end{array}$ & $\begin{array}{l}\text { HRS } \\
\text { HRS }\end{array}$ & $\begin{array}{l}\text { HRS } \\
\text { HRS }\end{array}$ & $\begin{array}{l}\text { SS } \\
\text { SS }\end{array}$ & $\begin{array}{l}\text { HRS } \\
\text { MRS }\end{array}$ \\
\hline
\end{tabular}

$N$ o t e. Bold figures indicate the change of strain susceptibility to antibiotics.

nem. Some changes of antibiotic sensitivity were found in 14 enterobacterial strains, resistant strains becoming sensitive ones and vice versa.

The Enterobacteriaceae changes from resistance to susceptibility $(R \rightarrow S)$ were found for the following antibiotics: amikacin (for one strain), cefazolin (for three strains), and tobramicin (for 1 strain).

Another variability type - from susceptibility to resistance $(S \rightarrow R)$ - was shown for the following antibiotics: amikacin ( 3 strains), chloramphenicol (1 strain), ciprofloxacin (3 strains), cefotaxime ( 2 strains), cefazolin (1 strain), ceftriaxone ( 2 strains), ceftazidime ( 3 strains), amoxicillin/clavulanic acid ( 3 strains). The enterobacterial variability index $(S \rightarrow R)$ is very important for patients' treatment, since any non-adequately chosen antibiotic does not promote successful therapy results.

Moderately susceptible enterobacterial strains demonstrate also the variability phenomenon becoming more resistant; at the same time, some susceptible strains of this bacterial group become moderately susceptible.

The data described above demonstrate the enterobacterial transition from to antibiotics susceptibility to antibacterial resistance and vice versa in the L41 cell line. We aimed to verify whether such phenomenon is ubiquitous enough and if it may be found also during Enterobacteriaceae cultivation in cell lines 


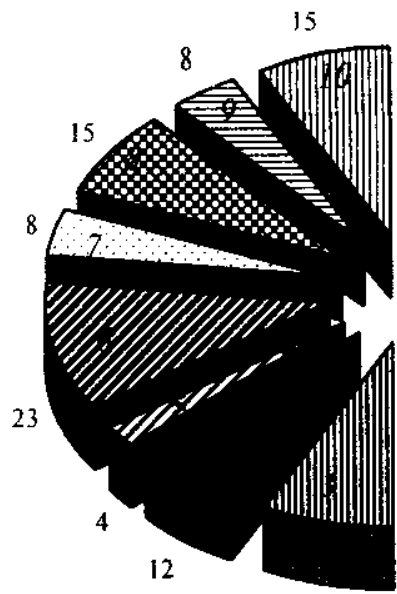

15

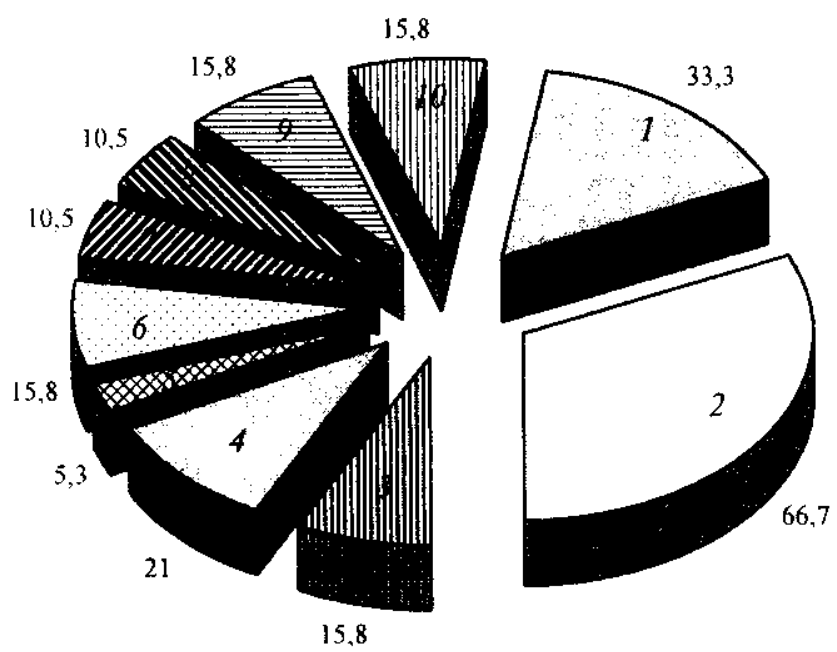

of other origin; so we used several of the abovementioned cell lines for their growth - RGNR (Fig. 1), Vero (Fig. 2), OH-1 (Fig. 3), and L41 (Fig. 4).

The analysis of the data presented proves the Enterobacteriaceae susceptibility to different antibiotic compounds does not depend on donor animal species used as primary culture. Seven enterobacterial strains changed their susceptibility/resistance following $24 \mathrm{~h}$ of their co-cultivation with eukaryotic cell, giving rise to both transitions $-(S \rightarrow R)$ and $(R \rightarrow$ $\rightarrow$ S). Cultivation of our strains in other four different cell lines revealed the same phenomenon.

The next step of our investigations was the susceptibility determination of a gram-positive bacterium, $S$. aureus, during its growth in the cell line L41. Analyzing these results, it should be noted that the antibiotic susceptibility profile changed only for a single $S$. aureus strain; the $(S \rightarrow R)$ transition was
Fig. 1. Sensitivity changes $(\%)$ to antibiotics $(I-10)$ following Enterobacteriaceae passages in RGNR cells: 1- stable; 2 - unstable; 3 - amk; 4- amp; 5 chl; 6-cip; $7-\mathrm{kf} ; 8-\mathrm{caz} ; 9-\mathrm{cro} ; 10-\mathrm{ctx}$

Fig. 2. Sensitivity changes $(\%)$ to antibiotics $(I-10)$ following Enterobacteriaceae passages in Vero cells: 1 - stable; 2 - unstable; 3 - amx; 4-amk; 5 caz; $6-\mathrm{ctx} ; 7-\mathrm{kf} ; 8-\mathrm{cip} ; 9-\mathrm{cro} ; 10-\mathrm{ctx}$

found for such antibiotic compounds as oxacillin, clindamycin, and cefazolin.

The data concerning the antibiotic susceptibility changes for yeast-like fungi (Candida genus representatives and $\mathrm{Cr}$. neoformans) are shown in Fig. 5 . The data obtained show that the $(S \rightarrow R)$ and $(R \rightarrow$ $\rightarrow$ S) transitions are found in seven Candida strains among 17 studied. The $(\mathrm{R} \rightarrow \mathrm{S})$ transition was detected for anti-Candida drugs: fluconazole (in 4 strains) and clotrimazole (in 2 strains). The transition $(\mathrm{S} \rightarrow \mathrm{R}$ ) was found for amphotericin B (in 5 strains), fluconazole (in 1 strain), and clotrimazole (in 1 strain).

Among two $\mathrm{Cr}$. neoformans strains studied here, the $(R \rightarrow S)$ transition for fluconazole was registered for a single strain.

These data indicate that the interactions between cultivated eukaryotic cells and parasitic ones including 

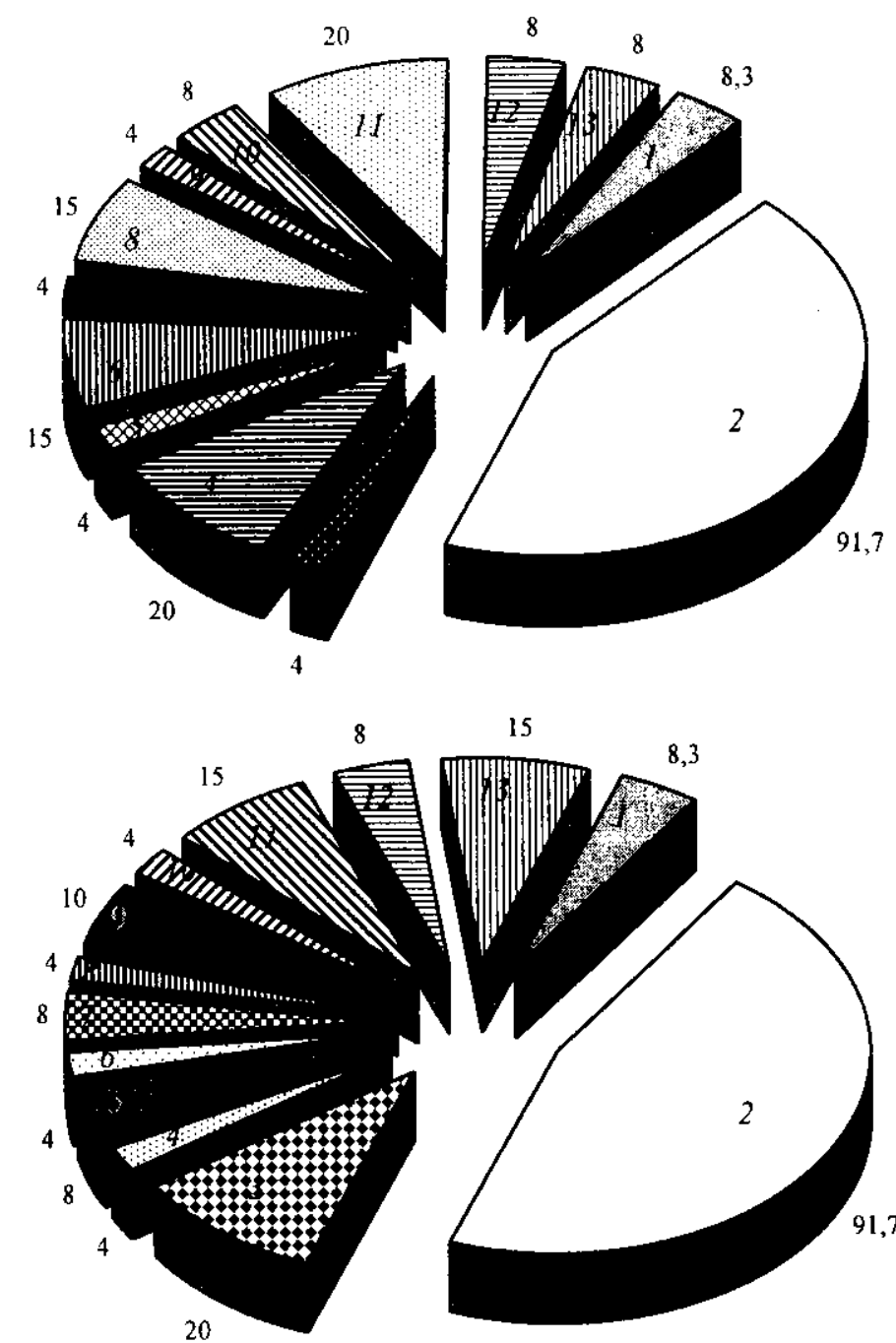

Fig. 3. Sensitivity changes $(\%)$ to antibiotics $(1-13)$ following Enterobacteriaceae passages in $\mathrm{OH}^{-1}$ cells: I-stable; 2 - unstable; 3 - amp; 4- amk; 5 amx; $6-\mathrm{ctx} ; 7-$ tet; $8-\mathrm{caz} ; 9-$ tob; $10-$ cip; $11-\mathrm{cro} ; 12-\mathrm{chl} ; 13-\mathrm{kf}$ gram-positive and gram-negative bacteria as well as yeast-like microorganisms cause both $(S \rightarrow R)$ and $(\mathrm{R} \rightarrow \mathrm{S})$ transitions. It leads to the conclusion the results of parasitic organism testing in nutrient media aimed at the determination of its susceptibility to antibiotics does not always reflect real antimicrobial effect in animal and human organisms, this situation being probably due to biofilm development on the monolayer surface. Therefore, we realized a series of experiments to determine the susceptibility of parasitic microorganisms in donor blood leukocytes, three passages of each microorganism studied having been carried out. We have found antibiotic susceptibility changes for differcnt strains of Enterobacteriaceae, $S$. aureus, $S$. epidermidis, $P$. aeruginosa, C. albicans, and $\mathrm{Cr}$. neoformans during their $2^{\text {nd }}-3^{\text {rd }}$ passages in human leukocytes.

Our comparative analysis of susceptibility to antibiotics in gram-positive and gram-negative bacteria as well as in yeast-like parasites during their passages through cultured human leukocytes shows the susceptibility changes; usually such changes are detected more often in gram-negative bacteria and yeast-like fungi.

Among 15 enterobacterial strains the susceptibility changes were registered in 12 ones. The $(S \rightarrow$ $\rightarrow R$ ) transitions were seen with such antibiotics as ciprofloxacin (for 1 strain), tetracycline (for 3 strains), and amikacin (for 1 strain). The $(R \rightarrow S)$ changes were registered for tobramycin (3 strains), ciprofloxacin (1 strain), amoxicillin/clavulanic acid (2 sträins), ceftazidine ( 2 strains), cefiriaxone (1 strain). The changes of MRS $\rightarrow$ HRS type were detected for cefazolin (2 strains), ciprofloxacin (2 strains), and amoxicillin/clavulanic acid (1 strain). The transition MRS $\rightarrow$ SS was found for tobramycin (in 1 strain), 

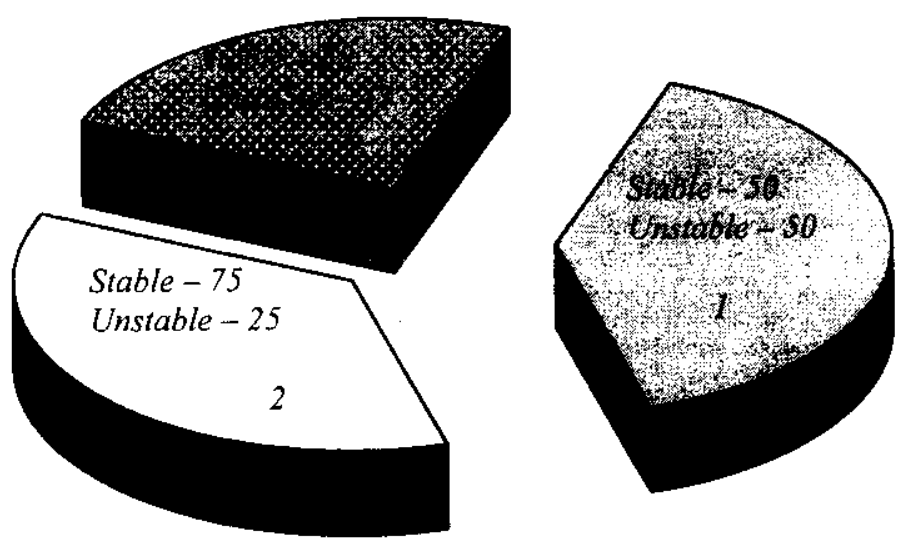

Fig. 5. Sensitivity changes $(\%)$ to antibiotics $(l-3)$ following Candido and Cryptococcus passages in cultivated cells: $1-$ flu; $2-$ clo; $3-$ amph amoxicillin/clavulanic acid (in 1 strain), and cefepime (in 1 strain). Only two ciprofloxacin-susceptible strains show the changes from SS to MRS phenotype.

The enterobacterial transitions from SS to resistant phenotype during the $2^{\text {nd }}-3^{\text {rd }}$ passages in human leukocytes occur in $80 \%$ of strains tested; they became resistant to tobramycin, ciprofloxacin, amoxicillin/clavulanic acid, ceftazidime, ceftriaxone, cefazolin, cefepime, and amikacin. The same bacteria keep their susceptibility unchanged as well as their resistance to ampicillin, gentamicin, and imipenem.

The data concerning the changes of $P$. aeruginosa susceptibility/resistance during the passages through human leukocytes show such changes in three strains among five ones. The transition from SS- to MRSphenotype was found for amikacin (2 strains) and netilmicin (1 strain). One strain susceptible to ciprofloxacin became resistant to this drug. The second $P$. aeruginosa strain with moderate resistance to cefoperazone lost this property becoming a susceptible strain. The third strain of high anti-amikacin resistance obtained a moderate resistant phenotype. One $P$. aeruginosa strain demonstrated unstable resistance to carbenicillin.

The susceptibility/resistance of $P$. aeruginosa strains concerning such antibiotics as gentamicin, imipenem, cefepime, azlocillin, and ceftazidime show no changes.

The passages of Candida genus representatives in human leukocyie cultures demonstrate often unstable type resistance against some antibiotics: fluconazole (in 2 strains), clotrimazole and amphotericin $B$ (in 1 strain); in addition, one Candida strain shows the HRS $\rightarrow$ MRS transition. Stable susceptibility to nystatin was found for all Candida strains used in our investigations.

Similar results were obtained with two $\mathrm{Cr}$. neoformans strains being constantly susceptible to nystatin during three passages. At the same time these passages sometimes lead to the resistance development in susceptible strains: one strain became resistant against clotrimazole and amphotericin B, another strain developed a fluconazole-resistant phenotype.

Two species of gram-positive bacteria $-S$. aureus (1 strain) and $S$. epidermidis (2 strains) - are less variable, the only transition (from higher to lower) was found for ciprofloxacin resistance.

It is evident that the changes in antibiotic susceptibility of different microorganisms appear following their passages through several cell lines of different species origin as well as through human leukocyte cultures. If the microorganisms change their susceptibility to certain antibiotics during passages, it is clear such drugs are not adequate for clinical practice use. Special attention should be paid to susceptibility changes in Enterobacteriaceae strains, $P$. aeruginosa, yeast-like organisms being susceptible to antibiotics chosen following the Bauer-Kirby test. Such changes may result in insufficient treatment results in clinical use. We found several antibiotics being continuously effective against Enterobacteriaceae and $P$. aeruginos $a$ - ampicillin, gentamicin, and imipenem.

The yeast-like microorganisms belonging to Candida and $\mathrm{Cr}$. neoformans change also their susceptibility to some antibiotics including fluconazole, clotrimazole, and amphotericin B, their susceptibility to nystatin being kept without changes. 
Table 2

Determination of enterobacterial strain 1646 susceptibility to antibiotics and its resistance to antibiotics during its growth in the L41 established cell line $(24 \mathrm{~h})$

\begin{tabular}{|c|c|c|c|c|c|c|c|c|c|c|c|c|c|c|c|}
\hline \multirow{2}{*}{ N Strains } & \multicolumn{15}{|c|}{ Antibiotic (the zone diameter, $\mathrm{mm}$, of inhibited growth for etalon strains? } \\
\hline & amp & let & gen & tob & amk & chl & cip & $\mathrm{ctx}$ & cpr & kf & cro & caz & $\operatorname{amx}$ & $\operatorname{imp}$ & cep \\
\hline 1646 & HRS & SS & HRS & MRS & MRS & HRS & SS & SS & SS & MRS & SS & SS & HRS & SS & SS \\
\hline $\mathrm{CC}$ & HRS & SS & HRS & SS & sS & HRS & SS & ss & ss & MRS & SS & SS & HRS & SS & SS \\
\hline $\mathrm{MCC}$ & SS & HRS & HRS & HRS & HRS & HRS & SS & HRS & SS & HRS & HRS & HRS & SS & MRS & HRS \\
\hline CMO & HRS & SS & HRS & MRS & MRS & HRS & SS & SS & SS & SS & SS & SS & HRS & SS & SS \\
\hline
\end{tabular}

Table 3

Influence of $\alpha-$ and $\gamma$-IFN on ability to antibiotics susceptibility changes

\begin{tabular}{|c|c|c|c|c|c|c|c|c|}
\hline \multirow{2}{*}{$\mathrm{N}$ Sirains } & \multirow{2}{*}{ Effecl } & \multicolumn{7}{|c|}{ Antibiotic (the zone diameter, $\mathrm{mm}$, of inhibited growih for etalon strains) } \\
\hline & & $a m x$ & ctx & cip & $\mathrm{kf}$ & caz & cro & $\mathrm{amk}$ \\
\hline \multirow[t]{3}{*}{724} & Initiation & sS & SS & SS & SS & SS & - & - \\
\hline & C & HRS & HRS & HRS & HRS & HRS & - & - \\
\hline & $\alpha$-IFN & HRS & HRS & HRS & HRS & HRS & - & - \\
\hline \multirow[t]{3}{*}{396} & Initiation & - & MRS & MRS & HRS & - & MRS & MRS \\
\hline & $\mathrm{C}$ & - & HRS & HRS & HRS & - & SS & HRS \\
\hline & $\gamma$-IFN & - & SS & HRS & MRS & - & SS & SS \\
\hline
\end{tabular}

The changes of microorganism susceptibility to antibiotics are due to numerous factors including properties of bacteria/yeast-like agents, properties of their host cells, culture media with all their components, and metabolic products. The unstable results obtained in our investigations and their changes during the passages carried out led us to a hypothesis concerning the metabolic products as the main variability factor. To verify this hypothesis, we carried out the following experiment in a model system using an enterobacterial strain, 1646, with multiple changes of susceptibility to antibiotics. The bacteria were grown in:

1. L41 cell culture washed carefully from its culture medium before bacterial infecting (cell culture, $\mathrm{CC}$ );

2. Medium following the L41 cell line cultivation (medium post cell cultivation, MCC);

3. RPMI-1640 (cell medium, original, CMO). The results obtained are presented in Table 2. They show the susceptibility changes to occur in the MCC containing cell metabolic products including also cytokines, e. g. IFN.

Due to these data, a question arose about possible IFN participation in changes of antibiotic sus- ceptibility for different microorganisms. To answer this question, we carried out some additional researches. The L41 cells were pre-treated by recombinant IFN preparations, IFN $-\alpha-2 b$, or by an IFN $-\gamma$ preparation (see above; both preparations contained $10^{5}$ I. U.) $\left(10^{8}\right.$ bacterial cells $\left./ \mathrm{ml}\right)$. The results obtained are given in Table 3.

The data presented in Table 3 show that during two strains $(724,396)$ strains cultivation in L4l cell line, they change their susceptibility to antibiotics, the susceptible bacteria becoming resistant to amoxicillin/clavulanic acid, cefotaxime, ciprofloxacin, ceftriaxone, and ceftazidime. The IFN- $c-2 b$ exerts no influence on susceptibility changes.

The results obtained in the experiments with the IFN- $y$ preparation concerning its effect on the strains 724 and 396 susceptibility/resistance suggest this cytokine to promote the $\mathrm{R} \rightarrow \mathrm{S}$ transition for amikacin, cefotaxime, ciprofloxacin, ceftriaxone, and cefazolin.

Similar results were obtained while cultivating Enterococcus faecalis in human neutrophils with gamma-IFN (1) and Listeria monocytogenes in macrophages with the same cytokine (2).

All these data allow the conclusion that the 
Bauer-Kirby test as well as other similar tests using bacterial nutrient media do not give information sufficient for therapeutic schema elaboration including antibiotic use in vivo. They need additional correction to determine real antibiotic susceptibility for different infectious agents. The cell-produced IFNs may exert their effect on bacteria ability to change their antibiotic susceptibility.

С. Л. Рибалко, О. R. Покас, В. А. Дєєв, Т. М. Лясковський, Т. М. Фурзікова, С. Т. Дядюн, Н. В. Іванська, Н. І. Настояща, П. П. Верхацький, С. Т. Сальков

Зміна чутливості до антибіотиків штамів бактерій і грибів при культивуваннні їх у перещеплюваних культурах клітин людини і тварин

Резюме

При взасмодї бактерій $i$ грибів 3 клітинами людини та тварин ідентифіковано феномен реверсії чутливості та резистентності до антибіотиків бактерій $i$ грибів. Властивість реверсї чутливості бактерій $і$ срибів до деяких антибіотиків нестабільна, вона змінюється від пасажу до пасаху, що може обумовлювати неефективність лікування антибіотиками при чинних методах встановлення чутливості до антибіотиків бактерій $i$ грибів. Автори пропонують модифікувати тест антибіотикочутливості бактерій $i$ грибів, виділених на поживних середовищах, культивуванням іх у культурі перещеплюваних клітин або лейкоцитах людини.

Ключові слова: антибіотики, клітини, ентеробактерії, резистентність.

С. Л. Рыбалко, Е. В. Покас, В. А. Деев, Т. М. Лясковский, Т. М. Фурзикова, С. Т. Дядюн, Н. В. Иванская, Н. И. Настоящая, ПI. П. Верхацкий, С. Т. Сальков

Изменение устойчивости к антибиотикам штаммов бактерий и дрожжеподобных грибов при культивировании их в перевиваемых культурах тканей человека и животных

Резюме

При взаимодействии бактерий и грибов с клетками человека и животных идентифицирован феномен реверсии чувствительности и резистентности к антибиотикам бактерий и грибов в зависимости от условий выращивания. Свойство реверсии чувствительности бактерий и грибов к некоторым антибиотикам нестабильно, оно изменяется от пассажа $к$ пассаху, что может обусловливать неэффективность лечения антибиотихами при существующем методе определения чувствительности к антибиотикам бактерий и грибов. Авторы предлагают модифицировать тест антибиотикочувствительно- сти бактерий и грибов, выделенных на питательных средах, культивированием их в культуре перевиваемьх клеток или лейкоцитах человека.

Ключевые слова: антибиотики, клетки, энтеробактерии, резистентность.

\section{REFERENCES}

1. Жалко-Титаренко В., Бондаренко Б. Н., Грисорьев А. Н. Динамика взаимодействия шигелл с эпителием в процессе заражения // Журн. микробиологии, эпидемиологии и иммунобиологии.-1986.-№ 4.-С. $21-24$.

2. Anvar Y., van Biesen $M$., Dasgupta $K$. Ineraction of biofilm bacteria with antibiotics in a novel in vitro chemostat system // Antimicrob. Agents Chemother.-1989.-33-P. 1824-1826.

3. Anvar $H$., Costerton $J$. W. Effective use of antibiotics in the treatment of biofilm-associated infections // ASM News.1992.-58. - P. 665-668.

4. Gilbert P., Collier J., Brown M. R. Influence of growth rate on susceptibility to antimicrobial agents: biofilms, cell cycle, dormancy, and stringent response // Antimicrob. Agents Chemother.-1990.-34.-P. 1865-1868.

5. Costerton J., Cheng K.-J. Geesey G. G., Anvar H. Bacterial biofilms in nature and disease $/ /$ Ann. Rev. Microbiol.1987.-41.-P. 435-464.

6. Gilbert $P$. Attachment and biofilm formation: the critical event in microbial pathogenesis // J. Pharm. Pharmacol.-1997.49, suppl. 4.-P. 8.

7. Голубев Д. Б., Соминина А. А., Медведева М. Н. Руководство по применению клеточных культур в вирусологии.-Ленинград: Медицина, 1976.-54 с.

8. National Committee for Clinical Laboratory Standards. Performance standards for antimicrobial susceptibility testing; eleventh informational supplement.--New York, 2001.--Vol. 2.$79 \mathrm{p}$.

9. Серия технических докладов ВО3 (критерии для интерпретации результатов испытаний, основанные на методе Бауэр-Кирби).-Женева, 1984.-N 673.-С. 147-169.

10. Onveji C. $O$., Nicolau D. P., Nightingale C. $H$., Bow $L$. Interferon-gamma effects on activities of gentamicin and vancomycin against Enterococcus faecalis resistant to the drugs: an in vitro study with human neutrophils // Int. J. Antimicrob. Agents.-1999.-11.-P. 31-37.

11. Ouadrhiri Y., Scorneaux B., Sibille Y., Tulkens P. M. Mechanism of the intracellular killing and modulation of antibiotic susceptibility of Listeria monocytogenes in THP-1 macrophages activated by gamma-interferon // Antimicrob Agents Chemother.-1999.-43.-P. 1242-1251.

12. Difco Manual // Dehydrated culture media and reagents for microbiology: Tenth edition. -Detroit, 1994.-P. 844-850.

удк 616.002.828: [577.181.5+62-581/584]+57.017.4 Надійшла до редакціi 31.01 .06 